\title{
EXCURSUS INTO THE HISTORY OF CALCULUS
}

\author{
S. S. KUTATELADZE
}

AbStRACT. This is a brief overview of some turning points in the history of infinitesimals.

The ideas of differential and integral calculus are traceable from the remote ages, intertwining tightly with the most fundamental mathematical concepts.

I admit readily that to present the evolution of views of mathematical objects and the history of the processes of calculation and measurement which gave an impetus to the modern theory of infinitesimals requires the Herculean efforts far beyond my abilities and intentions.

The matter is significantly aggravated by the fact that the history of mathematics has always fallen victim to the notorious incessant attempts at providing an apologia for all stylish brand-new conceptions and misconceptions. In particular, many available expositions of the evolution of calculus could hardly be praised as complete, fair, and unbiased. One-sided views of the nature of the differential and the integral, hypertrophy of the role of the limit and neglect of the infinitesimal have been spread so widely in the recent decades that it is impossible to ignore their existence.

It has become a truism to say (cf. [1]):

- The genuine foundations of analysis have for a long time been surrounded with mystery as a result of unwillingness to admit that the notion of limit enjoys an exclusive right to be the source of new methods.

However, Pontryagin was right to remark in [2, pp. 64-65] that

- In a historical sense, integral and differential calculus had already been among the established areas of mathematics long before the theory of limits. The latter originated as superstructure over an existent theory. Many physicists opine that the so-called rigorous definitions of derivative and integral are in no way necessary for satisfactory comprehension of differential and integral calculus. I share this viewpoint.

Considering the above, it is worthwhile to discuss a few turning points and crucial ideas in the evolution of analysis as expressed in the words of classics. The choice of the corresponding fragments is doomed to be subjective. Nevertheless, the selection below seems sufficient for anyone to acquire a critical attitude to the numerous incomplete and misleading delineations of the evolution of infinitesimal methods.

Key words and phrases. Differential, infinitesimal.

The Russian version of this talk appeared firstly in the mimeographed notes "Fundamentals of Nonstandard Mathematical Analysis" for the students of Novosibirsk State University in 1984. Its English versions served as introduction to Nonstandard Methods of Analysis by A. Kusraev and S. Kutateladze (Kluwer Academic Publisher, 1994) and Infinitesimal Analysis by E. Gordon, A. Kusraev, and S. Kutateladze (Kluwer Academic Publishers, 2002). 


\section{G. W. Leibniz And I. NeWTON}

The ancient name for differential and integral calculus is "infinitesimal analysis."'1]

The first textbook on this subject was published as far back as 1696 under the title Analyse des infiniment petits pour l'intelligence des lignes courbe. The textbook was compiled by de l'Hôpital as a result of his contacts with J. Bernoulli (senior), one of the most famous disciples of Leibniz.

The history of creation of mathematical analysis, the scientific legacy of its founders and their personal relations have been studied in full detail and even scrutinized. Each fair attempt is welcome at reconstructing the train of thought of the men of genius and elucidating the ways to new knowledge and keen vision. We must however bear in mind the principal differences between draft papers and notes, personal letters to colleagues, and the articles written especially for publication. It is therefore reasonable to look at the "official" presentation of Leibniz's and Newton's views of infinitesimals.

The first publication on differential calculus was Leibniz's article "Nova methodus pro maximis et minimis, itemque tangentibus, quae nec fractals nec irrationales quantitates moratur, et singulare pro illis calculi genus" (see [5]). This article was published in the Leipzig journal "Acta Eruditorum" more than three centuries ago in 1684 .

Leibniz gave the following definition of differential. Considering a curve $Y Y$ and a tangent at a fixed point $Y$ on the curve which corresponds to a coordinate $X$ on the axis $A X$ and denoting by $D$ the intersection point of the tangent and axis, Leibniz wrote:

- Now some straight line selected arbitrarily is called $d x$ and another line whose ratio to $d x$ is the same as of $\ldots y \ldots$ to $X D$ is called $\ldots d y$ or difference (differentia) ... of $y \ldots$

The essential details of the picture accompanying this text are reproduced in Fig. 1.

By Leibniz, given an arbitrary $d x$ and considering the function $x \mapsto y(x)$ at a point $x$, we obtain

$$
d y:=\frac{Y X}{X D} d x
$$

In other words, the differential of a function is defined as the appropriate linear mapping in the manner fully acceptable to the majority of the today's teachers of analysis.

Leibniz was a deep thinker and polymath who believed (see [7, pp. 492-493]) that

- the invention of the syllogistic form ranks among the most beautiful and even the most important discoveries of the human mind. This is a sort of universal mathematics whose significance has not yet been completely comprehended. It can be said to incarnate the art of faultlessness ....

Leibniz understood definitely that the description and substantiation of the algorithm of differential calculus (in that way he referred to the rules of differentiation) required clarifying the concept of tangent. He proceeded with explaining that

- we have only to keep in mind that to find a tangent means to draw the line that connects two points of the curve at an infinitely small distance, or the continued

\footnotetext{
${ }^{1}$ This term was used in 1748 by Leonhard Euler in Introductio in Analysin Infinitorum [3] (cf. 4 p. 324]).
} 
side of a polygon with an infinite number of angles which for us takes the place of the curve.

We may conclude that Leibniz rested his calculus on appealing to the structure of a curve "in the small."

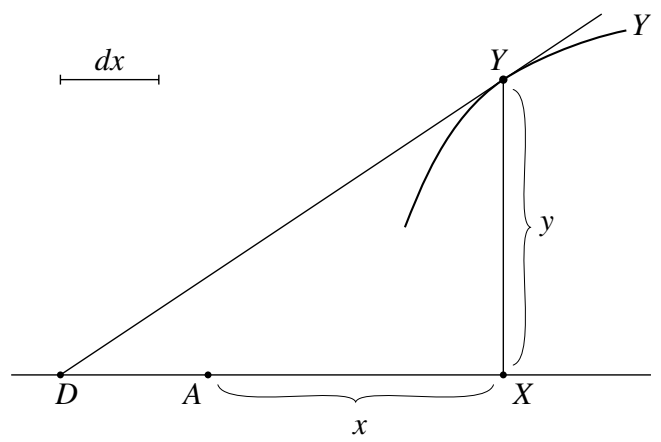

Fig. 1

At that time, there were practically two standpoints as regards the status of infinitesimals. According to one of them, which seemed to be shared by Leibniz, an infinitely small quantity was thought of as an entity "smaller than any given or assignable magnitude." Actual "indivisible" elements comprising numerical quantities and geometrical figures are the perceptions corresponding to this concept of the infinitely small. Leibniz did not doubt the existence of "simple substances incorporated into the structure of complex substances," i.e., monads. "It is these monads that are the genuine atoms of nature or, to put it short, elements of things" 6, p. 413].

For the other founder of analysis, Newton, the concept of infinite smallness is primarily related to the idea of vanishing quantities 8,9 . He viewed the indeterminate quantities "not as made up of indivisible particles but as described by a continuous motion" and "as increasing or decreasing by a perpetual motion, in their nascent or evanescent state."

The celebrated "method of prime and ultimate ratios" reads in his classical treatise Mathematical Principles of Natural Philosophy (1687) as follows (see [9, p. 101]:

- Quantities, and the ratios of quantities, which in any finite time converge continuously to equality, and before the end of that time approach nearer to each other than by any given difference, become ultimately equal.

Propounding the ideas which are nowadays attributed to the theory of limits, Newton exhibited the insight, prudence, caution, and wisdom characteristic of any great scientist pondering over the concurrent views and opinions. He wrote (see 8 , p. 169]):

- To institute an analysis after this manner in finite quantities and investigate the prime or ultimate ratios of these finite quantities when in their nascent state is consonant to the geometry of the ancients, and I was willing to show that in the method of fluxions there is no necessity of introducing infinitely small figures into geometry.

Yet the analysis may be performed in any kind of figure, whether finite or infinitely small, which are imagined similar to the evanescent figures, as likewise in 
the figures, which, by the method of indivisibles, used to be reckoned as infinitely small provided you proceed with due caution.

Leibniz's views were as much pliable and in-depth dialectic. In his famous letter to Varignion of February 2, 1702 9, stressing the idea that "it is unnecessary to make mathematical analysis depend on metaphysical controversies," he pointed out the unity of the concurrent views of the objects of the new calculus:

- If any opponent tries to contradict this proposition, it follows from our calculus that the error will be less than any possible assignable error, since it is in our power to make this incomparably small magnitude small enough for this purpose, inasmuch as we can always take a magnitude as small as we wish. Perhaps this is what you mean, Sir, when you speak on the inexhaustible, and the rigorous demonstration of the infinitesimal calculus which we use undoubtedly is to be found here....

So it can also be said that infinites and infinitesimals are grounded in such a way that everything in geometry, and even in nature, takes place as if they were perfect realities. Witness not only our geometrical analysis of transcendental curves but also my law of continuity, in virtue of which it is permitted to consider rest as infinitely small motion (that is, as equivalent to a species of its own contradictory), and coincidence as infinitely small distance, equality as the last inequality, etc.

Similar views were expressed by Leibniz in the following quotation (see 6] [ p. 190]) whose end in italics is often cited in works on infinitesimal analysis in the wake of Robinson [22, pp. 260-261]:

- There is no need to take the infinite here rigorously, but only as when we say in optics that the rays of the sun come from a point infinitely distant, and thus are regarded as parallel. And when there are more degrees of infinity, or infinitely small, it is as the sphere of the earth is regarded as a point in respect to the distance of the sphere of the fixed stars, and a ball which we hold in the hand is also a point in comparison with the semidiameter of the sphere of the earth. And then the distance to the fixed stars is infinitely infinite or an infinity of infinities in relation to the diameter of the ball. For in place of the infinite or the infinitely small we can take quantities as great or as small as is necessary in order that the error will be less than any given error. In this way we only differ from the style of Archimedes in the expressions, which are more direct in our method and better adapted to the art of discovery.

\section{L. Euler}

The 18th century is rightfully called the age of Euler in the history of mathematical analysis (cf. 13]). Everyone looking through his textbooks 14 will be staggered by subtle technique and in-depth penetration into the essence of the subject. It is worth recalling that an outstanding Russian engineer and scientist Krylov went into raptures at the famous Euler formula $e^{i \pi}=-1$ viewing it as the quintessential symbol of integrity of all branches of mathematics. He noted in particular that "here 1 presents arithmetic; $i$, algebra; $\pi$, geometry; and $e$, analysis."

Euler demonstrated an open-minded approach, which might deserve the epithet "systemic" today, to studying mathematical problems: he applied the most sophisticated tools of his time. We must observe that part and parcel of his research was the effective and productive use of various infinitesimal concepts, first 
of all, infinitely large and infinitely small numbers. Euler thoroughly explained the methodological background of his technique in the form of the "calculus of zeros." It is a popular fixation to claim that nothing is perfect and to enjoy the imaginary failures and follies of the men of genius ("to look for sun-spots" in the words of a Russian saying). For many years Euler had been incriminated in the "incorrect" treatment of divergent series until his ideas were fully accepted at the turn of the 20th century. We may encounter such a phrase in the literature: "As to the problem of divergent series, Euler was sharing quite an up-to-date point of view." It would be more fair to topsy-turvy this phrase and say that the mathematicians of today have finally caught up with some of Euler's ideas. In fact the opinion that "we cannot admire the way Euler corroborates his analysis by introducing zeros of various orders" is as self-assured as the statement that "the giants of science, mainly Euler and Lagrange, have laid false foundations of analysis." It stands to reason to admit once and for ever that Euler was in full possession of analysis and completely aware what he had created.

\section{G. Berkeley}

The general ideas of analysis greatly affected the lineaments of the ideological outlook in the 18th century. The most vivid examples of the depth of penetration of the notions of infinitely large and infinitely small quantities into the cultural media of that time are in particular Gulliver's Travels by Jonathan Swift published in 1726 (Lilliput and Brobdingnag) and the celebrated Micromegas 1752 written by bright and venomous F. M. Arouer, i.e., Voltaire. Of interest is the fact that as an epigraph for his classical treatise [22, Robinson chose the beginning of the following speech of Micromegas (cf. [10, p. 154]):

- Now I see clearer than ever that nothing can be judged by its visible magnitude. Oh, my God, who granted reason to creatures of such tiny sizes! An infinitely small thing is equal to an infinitely large one when facing you; if living beings still smaller than those were possible, they could have reason exceeding the intellect of those magnificent creatures of yours which I can see in the sky, and one foot of which could cover the earth.

A serious and dramatic impact on the development of infinitesimal analysis was made in 1734 by Bishop Berkeley, a great cleric and theologian, who published the pamphlet The Analyst, or a Discourse Addressed to an Infidel Mathematician, wherein it is examined whether the object, principles and inferences of the modern analysis are more deduced than religious mysteries and points of faith [11. By the way, this Infidel Mathematician was E. Halley, a brilliant astronomer and a young friend of Newton. The clerical spirit of this article by Berkeley is combined with aphoristic observations and killing precision of expression. The leitmotif of his criticism of analysis reads: "Error may bring forth truth, though it cannot bring forth science."

Berkeley's challenge was addressed to all natural sciences:

- I have no controversy about your conclusions, but only about your logic and method. How do you demonstrate? What objects are you conversant with, and whether you conceive them clearly? What principles you proceed upon; how sound they may be; and how you apply them? 
Berkeley's invectives could not be left unanswered by the most progressive representatives of the scientific thought of the 18th century, the encyclopedists.

\section{J. D'Alembert and L. Carnot}

A turning point in the history of the basic notions of analysis is associated with the ideas and activities of D'Alembert, one of the initiators and leading authors of the immortal masterpiece of the thought of the Age of Enlightenment, the French Encyclopedia or Explanatory Dictionary of Sciences, Arts, and Crafts.

In the article "Differential" he wrote: "Newton never considered differential calculus to be some calculus of the infinitely small, but he rather viewed it as the method of prime and ultimate ratios" [9, p. 157]. D'Alembert was the first mathematician who declared that he had found the proof that the infinitely small "do exist neither in Nature nor in the assumptions of geometricians" (a quotation from his article "Infinitesimal" of 1759).

The D'Alembert standpoint in Encyclopedia contributed much to the formulation by the end of the 18th century of the understanding of an infinitesimal as a vanishing magnitude.

It seems worthy to recall in this respect the book by Carnot Considerations on Metaphysics of the Infinitely Small wherein he observed that "the notion of infinitesimal is less clear than that of limit implying nothing else but the difference between such a limit and the quantity whose ultimate value it provides."

\section{B. Bolzano, A. Cauchy, and K. Weierstrass}

The 19th century was the time of building analysis over the theory of limits. Outstanding contribution to this process belongs to Bolzano, Cauchy, and Weierstrass whose achievements are mirrored in every traditional textbook on differential and integral calculus.

The new canon of rigor by Bolzano, the definition by Cauchy of an infinitely small quantity as a vanishing variable and, finally, the $\varepsilon-\delta$-technique by Weierstrass are indispensable to the history of mathematical thought, becoming part and parcel of the modern culture.

It is worth observing (see 9]) that, giving a verbal definition of continuity, both Cauchy and Weierstrass chose practically the same words:

An infinitely small increment given to the variable produces an infinitely small increment of the function itself.

CAUCHY

Infinitely small variations in the arguments correspond to those of the function.

WEIERSTRASS

This coincidence witnesses the respectful desire of the noble authors to interrelate the new ideas with the views of their great predecessors.

Speculating about significance of the change of analytical views in the 19th century, we should always bear in mind the important observation by Severi [12, p. 113] who wrote:

- This reconsideration, close to completion nowadays, has however no ultimate value most scientists believe in. Rigor itself is, in fact, a function of the amount 
of knowledge at each historical period, a function that corresponds to the manner in which science handles the truth.

\section{N. N. LUZIN}

The beginning of the 20th century in mathematics was marked by a growing distrust of the concept of infinitesimal. This tendency became prevailing as mathematics was reconstructed on the set-theoretic foundation whose proselytes gained the key strongholds in the 1930s.

In the first edition of the Great Soviet Encyclopedia in 1934, Luzin wrote (cf. [16, pp. 293-294]):

- As to a constant infinitely small quantity other than zero, the modern mathematical analysis, without discarding the formal possibility of defining the idea of a constant infinitesimal (for instance, as a corresponding segment in some nonArchimedean geometry), views this idea as absolutely fruitless since it turns out impossible to introduce such an infinitesimal into calculus.

The publication of the textbook Fundamentals of Infinitesimal Calculus by Vygodskil became a noticeable event in Russia at that time and gave rise to a serious and sharp criticism. Vygodskiu tried to preserve the concept of infinitesimal by appealing to history and paedeutics.

He wrote in particular (cf. [15, p. 160]):

- If it were only the problem of creating some logical apparatus that could work by itself then, having eliminated infinitesimals from considerations and having driven differentials out of mathematics, one could celebrate a victory over the difficulties that have been impeded the way of mathematicians and philosophers during the last two centuries. Infinitesimal analysis originated however from practical needs, its relations with the natural sciences and technology (and, later, with social sciences) becoming increasingly strong and fruitful in the course of time. Complete elimination of infinitesimals would hinder these relations or even make them impossible.

Discussing this textbook by Vygodskiu, Luzin wrote in the 1940s (cf. [16, p. 398]):

- This course, marked by internal integrity and lit by the great idea the author remains faithful to, falls beyond the framework of the style in which the modern mathematical analysis has been developed for 150 years and which is now nearing its completion.

Luzin's attitude to infinitesimals deserves special attention as apparent manifestation and convincing evidence of the background drama typical of the history of every profound idea that enchants and inspires the mankind. Luzin had a unique capability of penetration into the essence of the most intricate mathematical problems, and he might be said to possess a remarkable gift of foresight [17, 18, 21.

The concept of actual infinitesimals seemed to be extremely appealing to him psychologically, as he emphasized [16, p. 398]:

- The idea about them has never been successfully driven out of my mind. There are obviously some deeply hidden reasons still unrevealed completely that make our minds inclined to looking at infinitesimals favorably.

In one of his letters to Vygodskiu which was written in 1934 he predicted that "infinitesimals will be fully rehabilitated from a perfectly scientific point of view 
as kind of 'mathematical quanta.'" In another of his publications (cf. [19]), Luzin sorrowfully remarked:

- When the mind starts acquaintance with analysis, i.e., during the mind's spring season, it is the infinitesimals, which deserve to be called the "elements" of quantity, that the mind begins with. However, surfeiting itself gradually with knowledge, theory, abstraction and fatigue, the mind gradually forgets its primary intentions, smiling at their "childishness." In short, when the mind is in its autumn season, it allows itself to become convinced of the unique sound foundation by means of limits.

This limit conviction was energetically corroborated by Luzin in his textbook Differential Calculus wherein he particularly emphasized [20, p. 61]:

- To grasp the very essence of the matter correctly, the student should first of all made it clear that each infinitesimal is always a variable quantity by its very definition; therefore, no constant number, however tiny, is ever infinitely small. The student should beware of using comparisons or similes of such a kind for instance as "One centimeter is a magnitude infinitely small as compared with the diameter of the sun." This phrase is pretty incorrect. Both magnitudes, i.e., one centimeter and the diameter of the sun, are constant quantities and so they are finite, one much smaller than the other, though. Incidentally, one centimeter is not a small length at all as compared for instance with the "thickness of a hair," becoming a long distance for a moving microbe. In order to eliminate any risky comparisons and haphazard subjective similes, the student must remember that neither constant magnitude is infinitesimal nor any number, however small these might be. Therefore, it would be quite appropriate to abandon the term "infinitesimal magnitude" in favor of the term "infinitely vanishing variable," as the latter expresses the idea of variability most vividly.

\section{A. Robinson}

The seventh posthumous edition of this textbook by Luzin was published in 1961 simultaneously with Robinson's Non-Standard Analysis which laid a modern foundation for the calculus of infinitesimals. Robinson based his research on the local theorem by Mal'tsev, stressing its "fundamental importance for our theory" [22, p. 13] and giving explicit references to Mal'tsev's article dated as far back as 1936. Robinson's discovery elucidates the ideas of the founders of differential and integral calculus, witnessing the spiral evolution of mathematics.

\section{REFERENCES}

[1] Courant R. and Robbins G., What Mathematics Is. An Elementary Survey of Ideas and Methods [Russian translation], Prosveshchenie, Moscow (1967).

[2] Pontryagin L. S., Mathematical Analysis for Schoolchildren [in Russian], Nauka, Moscow (1980).

[3] Euler L., Introduction to Analysis of the Infinite. Book I [Russian translation], ONTI, Moscow (1936); [English translation], Springer-Verlag, New York etc. (1988).

[4] Kline M., Mathematical Thought From Ancient to Modern Times, Oxford University Press, Oxford (1972).

[5] 89 Leibniz G. W., "Nova Methodus pro Maximis et Minimis, Itemque Tangentibus, quae nec Fractals nec Irrationales Quattitates, et Singulare pro Illus Calculi Genns," Uspekhi Mat. Nauk, 3, No. 1, 166-173 (1948).

[6] Leibniz G. W., Selected Works. Vol. 1 [Russian translation], Mysl', Moscow (1983).

[7] Leibniz G. W., Selected Works. Vol. 2 [Russian translation], Mysl', Moscow (1984). 
[8] Newton I., The Mathematical Papers of Isaac Newton [Russian translation], ONTI, Moscow and Leningrad (1937).

[9] Reader on the History of Mathematics [in Russian], Prosveshchenie, Moscow (1977).

[10] Voltaire, Verses and Proses [Russian translation], Moskovskiŭ Rabochiı̌, Moscow (1997).

[11] Berkeley G., The Works. Vol. 1-4, Thoemmes Press, Bristol (1994).

[12] Severi F., "Italian algebraic geometry, its rigor, methods, and problems," Mathematics, 3, No. 1, 111-141 (1959).

[13] Boyer C. B., A History of Mathematics, John Wiley \& Sons Inc., New York etc. (1968).

[14] Euler L., Opera Omnia. Series Prima: Opera Mathematica. Vol. 1-29, Birkhäuser-Verlag, Basel etc.

[15] Vygodskiı M. Ya., Fundamentals of the Calculus of Infinitesimals [in Russian], GTTI, Moscow and Leningrad (1933).

[16] Luzin N. N., Collected Works. Vol. 3 [in Russian], Izdat. Akad. Nauk SSSR, Moscow (1959).

[17] Lavrent'ev M. A., "Nikolaŭ Nikolaevich Luzin," Uspekhi Mat. Nauk, 29, No. 5, 177-182 (1979).

[18] Lavrent'ev M. A., Science. Technical Progress. Personnel [in Russian], Nauka, Novosibirsk (1980).

[19] Vilenkin N., "The commander of 'Lusitania'," Znanie-Sila, No. 1, $27-29$ (1984).

[20] Luzin N. N., Differential Calculus [in Russian], Vysshaya Shkola, Moscow (1961).

[21] "Luzin N. N., the outstanding mathematician and teacher," Vestnik Akad. Nauk SSSR, No. 11, 95-102 (1984)

[22] Robinson A., Non-Standard Analysis, Princeton Univ. Press, Princeton (1996).

Sobolev Institute of Mathematics

4 Koptyug Avenue

Novosibirsk, 630090

RUSSIA

E-mail address: $\quad$ sskut@member.ams.org 